\title{
ORIGINAL ARTICLE \\ Postural bipedance in paraplegics under neuromuscular electrical stimulation: Is it possible to improve it based on sagittal spinal alignment?
}

\author{
RC Medeiros ${ }^{1,2,3}$, APB Jaccard ${ }^{1}$ and A Cliquet $\mathrm{Jr}^{1,4}$
}

Study design: Experimental trial based on the analytical study of radiographic standards of the sagittal spinal alignment in paraplegics in upright position under surface neuromuscular electrical stimulation (NMES).

Objectives: To evaluate changes in radiographic standards of the sagittal spinal alignment of paraplegics under three different models of NMES used to optimize the global bipedal posture.

Setting: The University Hospital Ambulatory (UNICAMP), Campinas, SP, Brazil.

Methods: Ten paraplegic patients were selected. Each patient underwent three different models of NMES. The influence that each NMES model exerted over the sagittal balance of the spine was evaluated by lateral panoramic X-rays. Wilcoxon's test was used to compare the modifications observed in each NMES model in the group studied.

Results: Using the femoral quadriceps muscles' NMES as the starting point, the inclusion of the gluteus maximus' NMES generated an increase of the lumbar lordosis and a decrease of the spinal tilt angle. These alterations resulted in partial improvement of the anterior sagittal imbalance. NMES of the paralyzed paravertebral lumbar muscles resulted in a more expressive increase on the lumbar lordosis, with no significant change on the spinal tilt. On the latter model, however, an improvement of $20 \%$ was observed in the global sagittal imbalance due to a posterior translation of the spine as pointed out by the decrease in the C7-HA horizontal distance.

Conclusions: The proposed NMES models were able to partially amend the anterior sagittal imbalance of the paraplegic patients in bipedal posture.

Spinal Cord (2012) 50, 309-314; doi:10.1038/sc.2011.123; published online 14 February 2012

Keywords: spinal cord injury; paraplegic standing; neuromuscular electrical stimulation; sagittal spinal alignment

\section{INTRODUCTION}

The body balance in neurologically normal people in the standing position is indicated by the segmental postural reflexes that are integrated in the spinal cord and modulated by higher neural centers. ${ }^{1}$ As two-thirds of the entire body mass is located above the hips, ${ }^{2}$ the main primary postural reflexes regulate the spinopelvic sagittal balance to maintain a close relationship between the gravity center and the perimeter of body support given by the feet, with minimum energy expenditure. ${ }^{3}$ The segmental postural reflexes below the area of spinal cord injury are absent in paraplegics. Potten et al. ${ }^{4}$ and Seelen et al. ${ }^{5}$ observed that, in these subjects, the loss of function of the erector spinal muscle is partially offset by the increase of the electromyographic activity and hypertrophy of the muscles latissimus dorsi and upper third of the trapezius. Several functional rehabilitation programs aim at improving the posture in paraplegics by stimulating the affected muscles. The simplest way to obtain the upright position in these patients is the open-loop neuromuscular electrical stimulation (NMES) of the knee extensors complemented by manual support. Matjacic and Bajd ${ }^{6}$ proposed a hands-free strategy to control the bipedal posture in paraplegics. Their theory was based on a mathe- matical model of an interconnected double-inverted pendulum that ignores the mobility and shape of the spine and considers the entire body segment above the hips as a single rigid structure. Unfortunately untill now no NMES system has been able to eliminate the need of manual support to keep the body balance and the human functional upright posture. More recently, Castro de Medeiros et al. ${ }^{7}$ described the radiographic parameters of sagittal spine balance in paraplegics in bipedal posture under NMES of knee extensors, with a significant anterior sagittal imbalance associated with an inverted pelvic tilt not compensated by any spinopelvic mechanism and counterbalanced by the upper limbs only. Currently, activation of lumbar paravertebral muscles in paraplegics has been used in two lines of research: the NMES, which has the goal of improving the functional performance of seated paraplegics, ${ }^{8}$ and the intercostal neurotization for the lumbar muscles. ${ }^{9}$ It is believed that in paraplegics, control of the spinopelvic balance is of paramount importance to obtain a functional bipedal posture. Hence, to understand the subject better, we described and compared the NMES spinopelvic radiographic changes of the main muscles involved in this process with those of the paraplegics in bipedal posture.

${ }^{1}$ Department of Orthopaedic Surgery, School of Medicine, Campinas State University (UNICAMP), Campinas, Brazil; ${ }^{2}$ Orthopaedia and Traumatology Group (GOT), São José Memorial Hospital, Recife, Brazil; ${ }^{3}$ Spine Surgery Group, Getúlio Vargas Hospital, Recife, Brazil and ${ }^{4}$ Department of Electrical Engineering, University of São Paulo (USP), São Carlos, Brazil

Correspondence: Dr RC Medeiros, Orthopaedia and Traumatology Group (GOT), São José Memorial Hospital, Av. Domingos Ferreira, 3856, apto 302, Boa Viagem, Recife, Pernambuco 52060 590, Brazil or Professor A Cliquet Jr, Department of Orthopaedic Surgery, School of Medicine, Campinas State University (UNICAMP), Campinas, Brazil. E-mail: castromed@hotmail.com or cliquet@fcm.unicamp.br

Received 21 June 2010; revised 16 September 2011; accepted 17 September 2011; published online 14 February 2012 


\section{MATERIALS AND METHODS}

Ten paraplegic male adult subjects were used, all with an ASIA (American Spinal Injury Association) Scale motor score of 50. Their neurological level ranged from T6 to T10, with car accident being their main cause of injury. Their average age was 36.6 years, average weight $81.6 \mathrm{~kg}$ and average sitting height $92.6 \mathrm{~cm}$. All of them had a history of more than 6 years of paraplegia and were involved in a rehabilitation program with NMES for at least 1 year (Table 2). Prior to the start of the study all subjects gave their written informed consent in accordance with the Helsinki Declaration and approved by the local ethics committee. Subjects were selected from the medical records and imaging exams among those actively participating in the NMES walking rehabilitation program at the Spinal Cord Injury Rehabilitation Ambulatory, Department of Orthopeedic and Traumatology, Campinas State University (Unicamp) Hospital, SP, Brazil. The inclusion criteria were: complete paralysis; absence of scoliosis and pelvic obliquity; absence of visual or vestibular comorbidities; and capacity of walking with NMES. Classical orthopedic physical examination discarded bone deformities and muscle shortening, and joint X-rays were obtained for exclusion of heterotopic ossification that limited the motion arcs of the hips and knees. The spasticity was assessed by applying the modified Ashworth Scale. The ASIA criteria were used to define the neurological level and to quantify the total residual neurological function. ${ }^{10}$ The seated height was measured from the ischium to the skull vertexwith the patient in the supine position, and his/her hips and knees flexed in $90^{\circ}$. Each patient underwent three sequential NMES electrode configurations (ECs): (1) quadriceps; (2) quadriceps+gluteus maximus; (3) quadriceps+gluteus maximus+paravertebral muscles below the injury and at $3 \mathrm{~cm}$ from the midline. NMES parameters were: steady voltage; $300 \mu$ s rectangular pulses; $25 \mathrm{~Hz}$ frequency; $70-150 \mathrm{~V}$ amplitude; and measured with a 1-kohm-load resistor. Ankle foot orthosis-type orthosis were used together with open loop transcutaneous NMES complemented by bimanual hand support. Clinically, all the subjects were plantigrade with extended knees. Aiming to optimize their fatigue endurance, all subjects underwent prior training of the muscle groups described above, twice a week for eight weeks.

Three lateral panoramic X-rays, one for each EC, were taken from all subjects to evaluate the eventual influences of the different NMES ECs on the sagittal spinal alignment in paraplegics. As a standard X-ray procedure, a long chassis with a film of $36.5 \mathrm{~cm} \times 91 \mathrm{~cm}$ was placed at a fixed distance of $230 \mathrm{~cm}$ from the radiation source, allowing exposure from the base of the skull to the proximal third of the femurs. Subjects were placed in a standing position with NMES complemented by a bimanual hand support so that the right shoulder was left toward the chassis and the face looking straight ahead. To reproduce the postural adjustments preceding the start of a paraplegic gait, a bimanual hand support was placed at variable distances and heights and adjusted to the balance needs of each subject. To keep the subjects in the same posture, the flexion angle of the upper limbs with the vertical axis of the body was set at $25^{\circ}$ as recommended by previous studies. ${ }^{11} \mathrm{~A}$ conventional goniometer was used while the X-rays were being taken. With the subjects standing under the EC 1, the NMES of the gluteus maximus and the lumbar paravertebral muscles was sequentially activated within short periods of time (average: 20 s) as necessary, just to obtain the X-rays. Each X-ray film was measured twice at different moments, with the averages used as the base for each patient. The resulting data were organized in three categories: (1) spine sagittal profile, (2) pelvis attitude and (3) spinopelvic balance (Figure 1 and Table 3). The kyphosis and the plumb line, previously found anterior to the upper posterior corner of S1, were represented by positive values, while negative values indicated lordosis and a plumb line posterior to the upper posterior corner of S1.

Epidemiological, anthropometric and clinical data were collected and are shown in Table 1. To define the neurological level and the quantification of the total residual neurological function, the ASIA criteria were used. ${ }^{10}$ All average values and s.d.'s were calculated from data generated in the three ECs of NMES. The Wilcoxon test was used to counterbalance the influence from the different ECs on the sagittal spine alignment, with the values being considered statistically significant when simultaneously presenting $T<8$ and $P<0.05$.

\section{RESULTS}

All X-ray measurements of sagittal alignment from upright paraplegic patients under the three different NMES ECs are shown in Table 3. Comparative analysis of the radiographic evaluation of the sagittal balance of the spine between the three NMES ECs suggests the following statistically significant observations (Table 4).

1. EC 1 compared with EC 2: The addition of NMES to the gluteus maximus caused posterior rotation of the pelvic tilt $\left(-4^{\circ}\right)$ and of the sagittal tilt $\left(-4.2^{\circ}\right)$, increased lordosis L5-S1 $\left(-3.9^{\circ}\right)$,

\section{Table 2 Available parameters on sagittal spine roentogenograms from subjects}

\begin{tabular}{|c|c|c|}
\hline $\begin{array}{l}\text { Anatomical format of } \\
\text { the spine }\end{array}$ & $\begin{array}{l}\text { Morphology and } \\
\text { kinetics of the pelvis }\end{array}$ & Spinopelvic balance \\
\hline $\begin{array}{l}\text { Thoracic kyphosis } \\
\text { (T4-T12) }\end{array}$ & Sacral slop (SS) & Horizontal distance (C7-S1) \\
\hline $\begin{array}{l}\text { Lumbar lordosis } \\
\text { (L1-L5) }\end{array}$ & Pelvic incidence (PI) & Horizontal distance $(\mathrm{C} 7-\mathrm{HA})$ \\
\hline $\begin{array}{l}\text { Lumbosacrum lordosis } \\
\text { (L1-S1) }\end{array}$ & Pelvic tilt (PT) & Horizontal distance (T4-HA) \\
\hline $\begin{array}{l}\text { Segmentar lumbar } \\
\text { lordosis: (L1-L2, } \\
\text { L2-L3, L3-L4, L5-S1) }\end{array}$ & $\begin{array}{l}\text { Pelvic radius } \\
\text { (PR-T12) }\end{array}$ & Horizontal distance (T4-L4) \\
\hline $\begin{array}{l}\text { Ratio: kyphosis } \\
\text { (T4-T12)/lordosis }\end{array}$ & $\begin{array}{l}\text { Sacrum-femur } \\
\text { angle (SFA) }\end{array}$ & Spinal tilt: horizontal line $\times$ C7-S1 \\
\hline
\end{tabular}

Sagittal tilt: T9-HA $\times$ vertical line Sacrum-spine angle: $\mathrm{SS} \times \mathrm{C} 7-\mathrm{S} 1$ Horizontal distance (T4-HA)

Abbreviations: $\mathrm{HA}$, hip axis; PR, pelvic radius.

Table 1 General characteristics of subjects

\begin{tabular}{|c|c|c|c|c|c|c|c|c|c|c|}
\hline Patient & 1 & 2 & 3 & 4 & 5 & 6 & 7 & 8 & 9 & 10 \\
\hline Gender & $M$ & $M$ & $\mathrm{M}$ & $M$ & $M$ & $M$ & $M$ & M & $M$ & $M$ \\
\hline Age (years) & 61 & 33 & 48 & 26 & 45 & 22 & 25 & 33 & 38 & 35 \\
\hline Mass (kg) & 74 & 91 & 84 & 73 & 95 & 72 & 90 & 75 & 84 & 78 \\
\hline Sitting height $(\mathrm{cm})$ & 86 & 89 & 87 & 96 & 97 & 92 & 96 & 93 & 94 & 96 \\
\hline Etiology & TB & FAP & Auto & FAP & Auto & Auto & Auto & Auto & Auto & Auto \\
\hline Neurological level & $\mathrm{T} 10$ & T7 & T8 & $\mathrm{T} 10$ & $\mathrm{~T} 10$ & T6 & T7 & T6 & T9 & T8 \\
\hline Total motor ASIA & 50 & 50 & 50 & 50 & 50 & 50 & 50 & 50 & 50 & 50 \\
\hline Period of neurological impairment (years) & 14 & 9 & 7 & 9 & 10 & 6 & 6 & 13 & 8 & 6 \\
\hline Time interval between impairment and NMES (years) & 11 & 1 & 5 & 5 & 9 & 1 & 5 & 3 & 4 & 3 \\
\hline Time interval of NMES (years) & 3 & 8 & 2 & 4 & 1 & 5 & 1 & 10 & 4 & 3 \\
\hline
\end{tabular}

Abbreviations: ASIA, American Spinal Injury Association; Auto, car crash; FAP, fire arms projectile; NMES, neuromuscular electrical stimulation; TB, vertebral tuberculosis. 

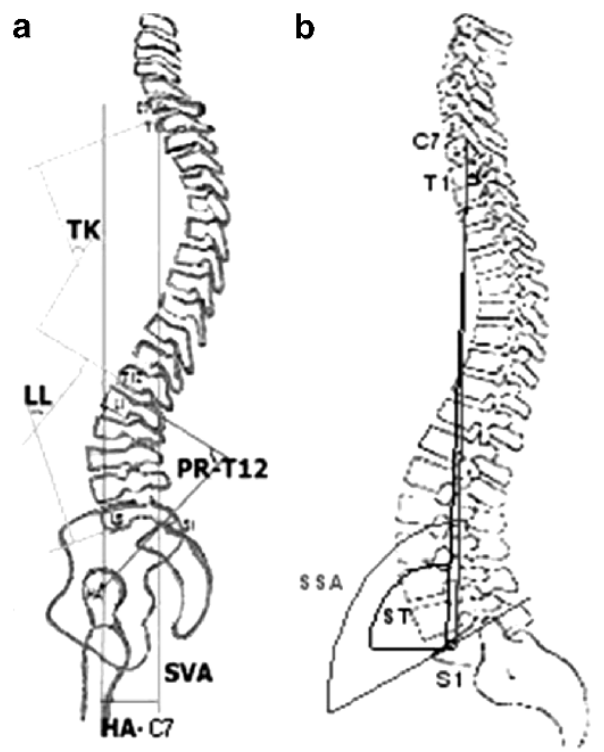

C

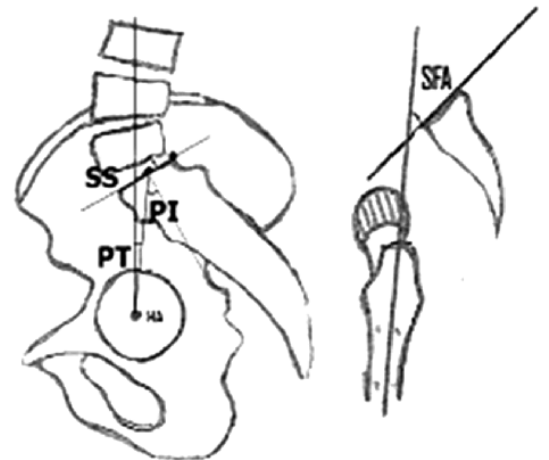

Figure 1 (a) Thoracic kyphosis (TK) T4-T12, lumbar lordosis (LL) L1-L5, Jackson's pelvic radius technique (PR-T12), horizontal distance C7 $\times$ hip-axis (HA-C7). (b) spinal tilt (ST), spinal-sacrum angle (SSA). (c) Sacral slop (SS), pelvic incidence (PI), pelvic tilt (PT). (d) Sacrum-femur angle (SFA).

Table 3 Roentogenographic measurements of sagittal alignment from upright paraplegic patients under three different models of neuromuscular electrical stimulation

\begin{tabular}{|c|c|c|c|c|c|c|}
\hline \multirow[t]{2}{*}{ Variables } & \multicolumn{2}{|c|}{ NMES model 1} & \multicolumn{2}{|c|}{ NMES model 2} & \multicolumn{2}{|c|}{ NMES model 3} \\
\hline & Mean & s.d. & Mean & s.d. & Mean & s.d. \\
\hline Thoracic kyphosis (T4-T12) & $35.8^{\circ}$ & $7.5^{\circ}$ & $36.7^{\circ}$ & $11.7^{\circ}$ & $37.6^{\circ}$ & $9.8^{\circ}$ \\
\hline Lumbar lordosis (L1-L5) & $-46.6^{\circ}$ & $4.9^{\circ}$ & $-45.9^{\circ}$ & $6.9^{\circ}$ & $-49.4^{\circ}$ & $6.3^{\circ}$ \\
\hline Lumbosacrum lordosis (L1-S1) & $-46.4^{\circ}$ & $5^{\circ}$ & $-45.1^{\circ}$ & $44.2^{\circ}$ & $-63.6^{\circ}$ & $3.5^{\circ}$ \\
\hline \multicolumn{7}{|l|}{ Segmentar lumbar lordosis } \\
\hline $\mathrm{L} 1-\mathrm{L} 2$ & $-8.5^{\circ}$ & $3.1^{\circ}$ & $-6.5^{\circ}$ & $2.9^{\circ}$ & $-7.4^{\circ}$ & $2^{\circ}$ \\
\hline L2-L3 & $-10.8^{\circ}$ & $1.9^{\circ}$ & $-8.6^{\circ}$ & $2.2^{\circ}$ & $-8.7^{\circ}$ & $2.7^{\circ}$ \\
\hline L3-L4 & $-15.7^{\circ}$ & $6.4^{\circ}$ & $-15^{\circ}$ & $4.2^{\circ}$ & $-13.6^{\circ}$ & $5.1^{\circ}$ \\
\hline L4-L5 & $-20.8^{\circ}$ & $6.1^{\circ}$ & $-22.8^{\circ}$ & $3.8^{\circ}$ & $-24.5^{\circ}$ & $5.9^{\circ}$ \\
\hline L5-S1 & $-20.5^{\circ}$ & $7.5^{\circ}$ & $-24.6^{\circ}$ & $7.1^{\circ}$ & $-22.2^{\circ}$ & $5.1^{\circ}$ \\
\hline Ratio kyphosis T4-T12/lordosis T12-PR & 0.3 & 0.1 & 0.4 & 0.1 & 0.4 & 0.1 \\
\hline Sacral slope (SS) & $60^{\circ}$ & $9.2^{\circ}$ & $59.2^{\circ}$ & $11.9^{\circ}$ & $57.4^{\circ}$ & $10.4^{\circ}$ \\
\hline Pelvic incidence (PI) & $45.4^{\circ}$ & $9.3^{\circ}$ & $47.1^{\circ}$ & $9.1^{\circ}$ & $42^{\circ}$ & $5.7^{\circ}$ \\
\hline Pelvic tilt (PT) & $15.9^{\circ}$ & $14^{\circ}$ & $11.9^{\circ}$ & $15^{\circ}$ & $9^{\circ}$ & $18.4^{\circ}$ \\
\hline Lumbopelvic lordosis PR-T12 & -97.8 & $10.2^{\circ}$ & $-99.1^{\circ}$ & $10.3^{\circ}$ & $-105.5^{\circ}$ & $11.9^{\circ}$ \\
\hline Horizontal distance (C7-S1), cm & 15.9 & 7.5 & 14.9 & 7.2 & 13.1 & 5.1 \\
\hline Horizontal distance $(\mathrm{C} 7-\mathrm{HA}), \mathrm{cm}$ & 19.5 & 10 & 17.2 & 10 & 15.7 & 8 \\
\hline Horizontal distance (T4-L4), cm & 8 & 6.5 & 7.4 & 6.4 & 6.9 & 5.9 \\
\hline Horizontal distance (T4-HA), cm & 14.1 & 11 & 11 & 11.2 & 10.8 & 8.6 \\
\hline Spinal tilt: Horizontal×C7—S1 & $74.3^{\circ}$ & $8.3^{\circ}$ & $75.7^{\circ}$ & $9.7^{\circ}$ & $75.6^{\circ}$ & $5.1^{\circ}$ \\
\hline Sacrum-spine angle: SS $\times$ C7-S1 & $18.4^{\circ}$ & $13.1^{\circ}$ & $13.8^{\circ}$ & $8.6^{\circ}$ & $13.6^{\circ}$ & $6.9^{\circ}$ \\
\hline Spinal tilt (T9-HA) & $12.7^{\circ}$ & $9.2^{\circ}$ & $8.5^{\circ}$ & $10.8^{\circ}$ & $8.4^{\circ}$ & $8.1^{\circ}$ \\
\hline
\end{tabular}

increased lordosis by the pelvic radius technique PR-T12 $\left(-1.3^{\circ}\right)$ and decreased the horizontal distance T4-HA $(-3.1 \mathrm{~cm})$.

2. EC 2 compared with EC 3: The addition of NMES to the paravertebral muscles decreased the distance C7-S1 $(-1.8 \mathrm{~cm})$ and increased lordosis L1-S1 $\left(-18.5^{\circ}\right)$.
3. EC 1 compared with EC 3: Simultaneous NMES of the gluteus maximus and lumbar paravertebral muscles increased lordosis PR-T12 $\left(-7.7^{\circ}\right)$, increased the sagittal tilt angle $\left(-4.3^{\circ}\right)$, decreased the distance $\mathrm{C} 7-\mathrm{HA}(-3.8 \mathrm{~cm})$ and T4-HA $(-3.3 \mathrm{~cm})$ and decreased the T4-T12/T12-PR ratio. 
Table 4 Wilcoxon test and the influence of different models of electric stimulation on the sagittal profile of the spine

\begin{tabular}{|c|c|c|c|c|c|c|}
\hline \multirow[t]{2}{*}{ Variables } & \multicolumn{6}{|c|}{ Measurements } \\
\hline & \multicolumn{2}{|c|}{$R \times 1 \times R \times 2$} & \multicolumn{2}{|c|}{$R \times 1 \times R \times 3$} & \multicolumn{2}{|c|}{$R \times 2 \times R \times 3$} \\
\hline Horizontal distance (C7-S1), cm & 9.5 & $>0.05$ & 9.0 & $>0.05$ & 7.5 & $<0.05$ Sig. \\
\hline Thoracic kyphosis (T4-T12) & 21.0 & $>0.05$ & 19.0 & $>0.05$ & 22.0 & $>0.05$ \\
\hline Sagital tilt $(\mathrm{T} 9-\mathrm{HA})$ & 5.0 & $<0.05$ Sig. & 3.0 & $<0.05$ Sig. & 26.5 & $>0.05$ \\
\hline Sacral slop (SS) & 20.5 & $>0.05$ & 9.5 & $>0.05$ & 14.5 & $>0.05$ \\
\hline Pelvic incidence $(\mathrm{PI})$ & 24.5 & $>0.05$ & 9.0 & $>0.05$ & 8.0 & $<0.05$ Sig. \\
\hline Pelvic tilt (PT) & 5.0 & $<0.05$ Sig. & 23.0 & $>0.05$ & 15.0 & $>0.05$ \\
\hline Sacrum-spine angle: $\mathrm{SS} \times \mathrm{C} 7-\mathrm{S} 1$ & 27.5 & $>0.05$ & 16.5 & $>0.05$ & 19.0 & $>0.05$ \\
\hline Horizontal distance (C7-HA), cm & 8.5 & $>0.05$ & 6.0 & $<0.05$ Sig. & 9.0 & $>0.05$ \\
\hline L3-L4 & 27.0 & $>0.05$ & 25.0 & $>0.05$ & 15.0 & $>0.05$ \\
\hline L4-L5 & 18.5 & $>0.05$ & 8.5 & $>0.05$ & 18.0 & $>0.05$ \\
\hline L5-S1 & 3.0 & $<0.05$ Sig. & 22.5 & $>0.05$ & 20.0 & $>0.05$ \\
\hline L4-S1 & 11.5 & $>0.05$ & 14.0 & $>0.05$ & 17.5 & $>0.05$ \\
\hline Lumbopelvic lordosis PR-T12 & 0.0 & $<0.05$ Sig. & 6.0 & $<0.05$ Sig. & 42.0 & $>0.05$ \\
\hline Horizontal distance (T4-HA), $\mathrm{cm}$ & 3.0 & $<0.05$ Sig. & 7.0 & $<0.05$ Sig. & 25.0 & $>0.05$ \\
\hline Horizontal distance (T4-L4), cm & 21.0 & $>0.05$ & 17.0 & $>0.05$ & 26.0 & $>0.05$ \\
\hline Ratio kyphosis T4-T12/lordosis T12-PR & 9.0 & $>0.05$ & 4.0 & $<0.05$ Sig. & 14.0 & $>0.05$ \\
\hline Spinal tilt: Horizontal $\times C 7-S 1$ & 18.0 & $>0.05$ & 19.0 & $>0.05$ & 27.0 & $>0.05$ \\
\hline Sacrum-spine angle: $\mathrm{SS} \times \mathrm{C} 7-\mathrm{S} 1$ & 22.0 & $>0.05$ & 23.0 & $>0.05$ & 21.0 & $>0.05$ \\
\hline
\end{tabular}

Abbreviation: Sig., statistically significant.

\section{DISCUSSION}

Studies on healthy volunteers describe the spinopelvic interrelations as major controllers of the spine sagittal balance and of the body gravity line. ${ }^{12-15}$ Schwab et al. ${ }^{15}$ analyzed the sagittal balance and the gravity line and concluded that every spinopelvic compensation has as its ultimate goal of maintaining the gravity center in close relationship with the feet positioning. According to Van der Spek et al. ${ }^{16}$ the degree of stabilization of the hips and the distance from the position of crutches to support paraplegics lacks statistically significant influence both on the posture adopted by patients and on the force applied to the crutches although it allows a stable orthostatic posture. These authors still suggest that the flexibility of the spine and the effect of the lumbar paravertebral muscles are fundamental to postural stability and load bearing and that the new study models should include these variables to optimize the control of the postural balance. Analyzing the sagittal spinal balance of spine-injured patients with NMES applied to the quadriceps, Medeiros et al. ${ }^{7}$ described a pattern characterized by apparently fixed flexion of the hips, sagittal curvatures of the spine presenting 'normal values', but not offsetting the pelvic effects and large anterior sagittal imbalance that was supported by the upper limbs. In this study, all patients submitted to NMES of just the quadriceps (EC 1) showed the sagittal pattern described above (Figure 2a).

In an attempt to optimize the position of the paraplegics' gravity line seen in the EC 1, we proposed two experimental models of NMES that aim to reproduce the spinopelvic compensations described in the literature.
Lieberman et al. ${ }^{11}$ reiterated that the anatomy of the thoracolumbar fascia/erector spinae/gluteus maximus work together to achieve extension of the spine. In addition, it is known that previous sagittal imbalances are usually offset by retroversion of the pelvis (pelvic tilt) from the extension of the hips. However, according to Roussouly et al. ${ }^{14}$ no correlation was found between the positions of the C7 plumb line and the line of body gravity, the latter being related to the spinal tilt, regardless of the value of the sacral slope. To push back the anterior sagittal imbalance observed earlier in patients undergoing NMES EC 1, we applied NMES to the bilateral gluteus maximus (EC 2 ) in order to roll back both the pelvic and the spinal tilts. The NMES of the gluteus maximus decreased the sagittal tilt and, a $22 \%$ average improvement over previous sagittal imbalances represented the decrease in the horizontal distance T4-HA (Figure 2b). This may have resulted from a higher tension generated on the thoracolumbar fascia exerted by the contraction of the gluteus maximus.

In EC 3, NMES was added to the paralyzed paravertebral lumbar muscles to increase lumbar lordosis, obtain maximum rollback of the spinal tilt and to improve the positioning of the gravity line. In this situation lumbosacrum lordosis increased $18.5^{\circ}$ on average (relative to EC 2) with rollback of the sagittal tilt and $3.8 \mathrm{~cm}$ posterior translation (19.4\%) of the C7-HA horizontal distance from EC 1 (Figure 2c).

The electroneuromyography by Lyons et al. ${ }^{17}$ in healthy volunteers showed that the hip extension moment results from the contribution of the gluteus maximus' lower portion+hamstrings+adductor magnus. Németh and Ohlsén. ${ }^{18}$ found that the moment arm of gluteus maximus decreased when increasing the hip flexion angle. 
a

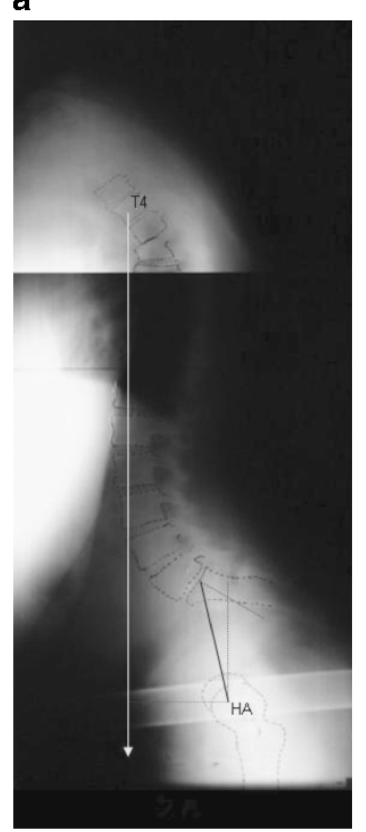

b

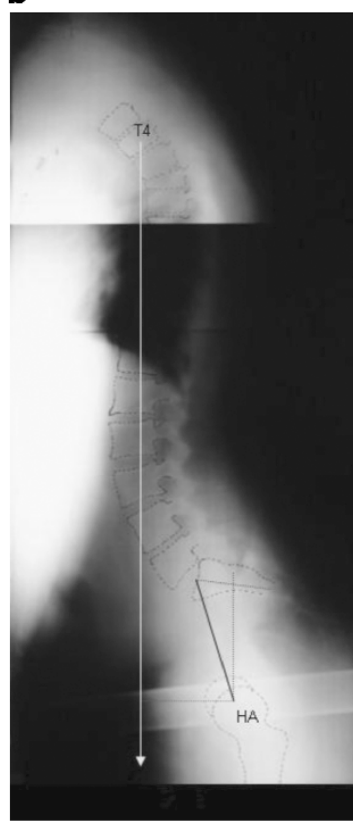

c

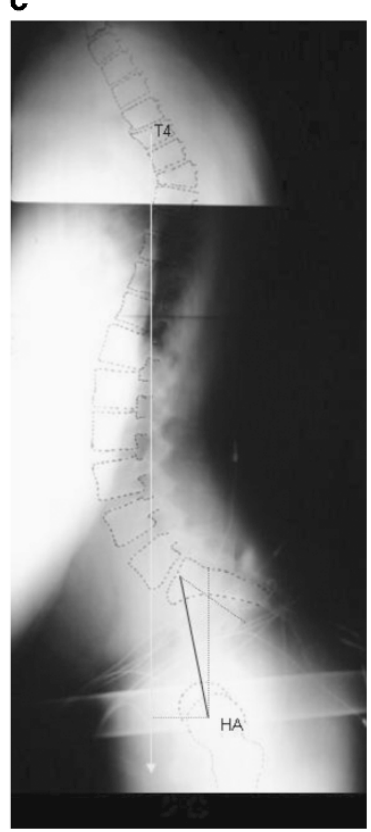

Figure 2 Case 6 was stimulated with three different models of neuromuscular electrical stimulation (NMES). Note a progressive increase in lumbar lordosis and a decrease in sagittal imbalance (T4-HA) with the add on NMES channels. (a) Femoral quadriceps NMES. (b) Femoral quadriceps+gluteus maximus NMES. (c) Femoral quadriceps+gluteus maximus+paravertebral lumbar NMES.

The hamstrings showed an increase in the moment arm length up to an average of $35^{\circ}$ in hip flexion and then a decrease with increase in hip flexion angle. For the adductor magnus, the moment arm showed an increase up to $75^{\circ}$ and then a decrease. In all three NMES models the patients showed positive sacrum-femur angles, indicating flexed hips. We expected that the gluteus maximus' NMES would be capable of extending the hips. We believe that it was not possible because of the mechanical disadvantage of short-extension lever arm generated by the gluteus maximus' NMES to offset the long flexing lever arm generated by the summation of the spinal length+anterior sagittal imbalance+contraction of the rectus femoralis' hip-flexor portion.

The NMES configuration used was unable to correct the paraplegics' spine sagittal imbalance. However, one can suggest with some criticism that, future NMES ECs should include complementary channels to the hamstrings aiming at optimizing the hips' extension lever arm in such a manner, so as to translate the spine by the rollback of the pelvic tilt. Sixty percent of the studied subjects subjectively reported an important decrease in the strength exerted by the upper limbs while in bipedal posture under the NMES EC 3. Even though this was not measured, such a fact could have a major impact on the incidence of late shoulder dysfunction because of the overloading of the upper limbs as described in the literature. ${ }^{19}$

\section{CONCLUSION}

The proposed NMES ECs applied to paraplegic patients on bipedal posture allowed a partial correction of the anterior sagittal imbalance. The major compensating mechanisms observed were: (1) posterior rotation of the sagittal tilt caused by the increase of the lumbopelvic lordosis and (2) posterior translation of C7 done by the paralyzed lumbar NMES. Despite of the statistically significant result obtained in this study, the same cannot be said in terms of a significant clinical improvement specific to the posterior rotation of the pelvic tilt promoted by the hips' extension through isolated bilateral NMES on the gluteus maximus.

\section{CONFLICT OF INTEREST}

The authors declare no conflict of interest.

1 Morningstar MW, Pettibon BR, Schlappi H, Schilappi M, Ireland TV. Reflex control of the spine and posture: a review of the literature from a chiropractic perspective. Chiropractic \& Osteopathy 2005; 13: 16

2 Konz RJ, Fatone S, Stine RL, Ganju A, Gard SA, Ondra SL. A kinematic model to assess motion during walking. Spine 2006; 24: E898-E906.

3 Saha D, Gard S, Fatone S. The effect of trunk flexion on able-bodied gait. Gait Posture 2007; 27: 653-660.

4 Potten YJM, Seelen HAM, Drukker J, Reulens JPH, Drost MR. Postural muscle responses in the spinal cord injured persons during forward reaching. Ergonomics 1999; 42: 1200-1215

5 Seelen HAM, Potten YJM, Drukker J, Reulens JPH, Pons C. Development of new muscle synergies in postural control in spinal cord injured subjects. J Electromyogr Kinesiol 1998; 8: 23-34.

6 Matjacic Z, Bajd T. Arm-free paraplegic standing - part II: Experimental results. IEEE Trans Rehab Eng 1998; 6: 139-150.

7 Castro de Medeiros R, Jaccard APB, Cliquet A. Sagittal spinal alignment in paraplegics: a new paradigm for the rehabilitation under neuromuscular electrical stimulation. Spinal Cord 2010; 48: 251-256.

8 Kukke SN, Triolo R. The effects of trunk stimulation on bimanual seated workspace. IEEE Trans Rehab Eng 2004; 12: 177-185.

9 Zhang S, Johnston L, Zhang Z, Ma Y, Hu Y, Wang J et al. Restoration of stepping-forward and ambulatory function in patients with paraplegia: rerouting of vascularized intercostals nerves to lumbar nerve roots using selected interfascicular anastomosis. Surg Technol Int 2003; 11: 244-248.

10 Maynard Jr FM, Brackem MB, Creasey G, Ditunno Jr JF, Donovan WH, Ducker TB et al. International standards for neurological and functional classification on spinal cord injury patients (revised). Spinal Cord 1997; 35: 266-274.

11 Lieberman DE, Raichlen DA, Pontzer H, Bramble DM, Cutright-Smith E. The human gluteus maximus and its role in running. J Exp Biol 2006; 209: 2143-2155.

12 Jackson RP, Peterson MD, McMannus AC, Hales C. Compensatory spinopelvic balance over the hip axis and better reliability in measuring lordosis to the pelvic radius on standing lateral radiographs of adult volunteers and patients. Spine 1998; 23: 17501767 
13 Legaye J, Duval-Beaupère G, Hecquet J, Marty C. Pelvic incidence: a fundamental pelvic parameter for three-dimensional regulation of spinal sagittal curves. Eur Spine $J$ 1998; 7: 99-103.

14 Roussouly P, Gollogly S, Noseda O, Berthonnaud E, Dimnet J. The vertical projection of the sum of the ground reactive forces of a standing patients is not the same as the C7 plumb line (A radiographic study of the sagittal alignment of 153 asymptomatic volunteers). Spine 2006; 11 . E320-E325.

15 Schwab F, Lafage V, Boyce R, Skalli W, Farcy J-P. Gravity line analysis in adult volunteers. Spine 2006; 25: E959-E967.
16 van der Spek JH, Veltink PH, Hermens HJ, Koopman BFJM, Boom HBK. Static and dynamic evaluation of the influence of supplementary hip-joint stiffness on crutchsupported paraplegic stance. IEEE Trans Rehab Eng 2003; 11: 452-462.

17 Lyons K, Perry J, Gronley JK, Barnes L, Antonelli D. Timing and relative intensity of hip extensor and abductor muscle action during level and stair ambulation: an EMG study. Phys Ther 1983; 63: 1597-1605.

18 Németh G, Ohlsén $\mathrm{H}$. In vivo moment arm lengths for hip extensor muscles at different angles of hip flexion. J Biomech 1985; 18: 129-140.

19 Baley JS, Cochran TP, Sledge CB. The weight bearing shoulder: the impingement syndrome in paraplegics. J Bone Joint Surg 1987; 62A: 676-678. 